\title{
The Impact of Rurality, Underlying Cardiovascular Disease, and Socioeconomic Vulnerability on COVID-19 Outcomes in Georgia
}

\author{
Michael Morgan, $\mathrm{BS}^{1^{*}}$, Lavannya Atri, $\mathrm{MS}^{1^{*}}$, Jennifer L. Waller, $\mathrm{PhD}^{1,2}$, D. Douglas Miller, MD, CM, MBA ${ }^{1,2,3,4}$, and Adam E. \\ Berman, MD, MSc, $\mathrm{MPH}^{1,2,3,4}$ \\ ${ }^{1}$ Medical College of Georgia, Augusta, GA; ${ }^{2}$ Department of Population Health Science, Medical College of Georgia, Augusta, GA; ${ }^{3}$ Department \\ of Medicine, Medical College of Georgia, Augusta, GA; and ${ }^{4}$ Institute for Public and Preventive Health, Augusta University, Augusta, GA. \\ "Indicates co-primary authorship of this manuscript.
}

Corresponding Author: Michael Morgan • Medical College of Georgia 2717 Wicklow Drive, Augusta, GA. 30909 • Telephone: (770) 877-1375 • Fax: (706) 721-5150•Email: mimorgan@augusta.edu

\section{ABSTRACT}

Background: COVID-19 related illnesses have been associated with an increased prevalence of cardiovascular disease sequelae and worsened socioeconomic variables. We sought to investigate the relationship between COVID-19 outcomes, underlying cardiovascular disease, and socioeconomic determinants of health in rural and non-rural counties in the state of Georgia.

Methods: COVID-19, demographic, and socioeconomic data were acquired from publicly available databases including the Centers for Disease Control and Prevention's Social Vulnerability Index. The relationship between COVID-19 outcomes and markers of cardiovascular disease burden, rurality, and socioeconomic determinants of health was assessed at the county level in Georgia through the beginning of August 2020 using univariable and multivariable Poisson regression modeling.

Results: In adjusted models, the risk of COVID-19 incidence was significantly higher in residents of non-rural Georgia counties while we observed no significant difference in COVID-19 case-fatality rates between residents of rural and non-rural Georgia counties. A significant adverse association between risk of COVID-19 cumulative case-fatality rates and recent mortality rates of stroke was detected, while counties with historically higher coronary heart disease death rates demonstrated significantly lower risk ratio of COVID-19 cumulative case-fatality rates. Additionally, Georgia counties with worsened indices of social and economic vulnerability demonstrated significantly higher risk ratio of COVID-19 incidence and case-fatality rates.

Conclusions: In Georgia, COVID-19 incidence is adversely associated with non-rural county status, while both incidence and case-fatality rates are associated with historical indices of cardiovascular disease outcomes and higher social vulnerability. Efforts to mitigate COVID-19 spread and improve COVID-19 outcomes in Georgia may require additional focus on these most vulnerable areas.

Keywords: COVID-19, SARS-CoV-2, cardiovascular disease, socioeconomic determinants of health, rural health.

\section{INTRODUCTION}

A significant proportion of patients affected by COVID-19 have pre-existing cardiovascular comorbidities (Guzik et al., 2020; Zheng et al., 2020). These patients have been found to exhibit a higher risk of progression to more severe COVID-19 infection (Paramasivam et al., 2020; Wang et al., 2020; Zheng et al., 2020). COVID-19's pro-inflammatory effects on the cardiovascular system have been shown to be mediated by its interaction with endothelial cell ACE2 receptors leading to potential widespread vascular damage, myocardial injury, and cardiac arrhythmias (Bansal, 2020; Huertas, 2020; Madjid et al., 2020; Varga et al., 2020). In addition to populations with cardiovascular comorbidities, rural communities face an increased risk of a higher case-fatality ratio from COVID-19 compared to non-rural communities (Kaufman et al; Lakhani et al., 2020). This risk may be due to rural areas' aging demographics, lower socioeconomic resources, reduced access to care, and higher rates of underlying chronic health conditions such as cardiovascular disease (CVD) when compared to their non-rural counterparts (Henning-Smith et al, 2020; Kaufman et al, 2020; Peters, 2020; Souch and Cossman, 2020). Socially vulnerable populations are another group disproportionately affected by COVID-19 due to structural inequities in housing, nutrition, income, education, and environment (Holuka et al., 2020; Karaye and Horney, 2020).

Due to the recent onset of the COVID-19 pandemic, relationships between cardiovascular disease, rurality, social vulnerability, and COVID-19 disease severity are still emerging. We sought to better describe the relationship between our dependent variables of COVID-19 incidence and case-fatality rates with exposures such as socioeconomic indices of health, rurality, and chronic CVD burden across Georgia's counties through the beginning of 
August 2020. This was achieved by utilizing Georgia Department of Public Health and CDC databases to accumulate county-level data for the aforementioned variables. Socioeconomic indices of health included factors such as socioeconomic status, household composition, minority status, and housing/transportation. County rurality status was defined by the Georgia Department of Public Health OASIS database, and the chronic CVD burden was assessed by obtaining fatality rates of coronary heart disease, heart failure, hypertension, stroke, and cardiac dysrhythmias per 100,000.

\section{METHODS}

In this ecological study, county-level COVID-19 incidence and mortality rates were retrospectively obtained from the Georgia Department of Public Health Daily Status Report from the beginning of the pandemic through August 1, 2020 (Georgia Department of Public Health, 2020). Rurality status was obtained via the Georgia Department of Public Health OASIS database (Georgia Department of Public Health, 2020). 2016-2018 cardiovascular outcomes data for all 159 Georgia counties was obtained from the CDC database and included all forms of cardiovascular causes of death expressed as number per 100,000 (Centers for Disease Control and Prevention, 2020).

The US Centers for Disease Control and Prevention's (CDC) Social Vulnerability Index (SVI) is a measure of a community's susceptibility to adverse health impacts during times of emergency such as disease outbreak (Centers for Disease Control and Prevention, 2018) and this index was used to help quantify socioeconomic variables across all Georgia counties. The SVI is composed of four themes: socioeconomic status, household composition, minority status, and housing/transportation. This comprehensive assessment tool allows for more granular assessment of a community's potential vulnerability in a public health crisis. 2018 CDC SVI data were used as a measure of social vulnerability (Centers for Disease Control and Prevention, 2018). Each Georgia county was ranked for each of the four main themes, ranging from 0 to 100 , with 0 being the least vulnerable county in the state and 100 being the most vulnerable county in the state. In addition to the SVI, other indices of economic well-being have been described. The Distressed Community Index (DCI), a novel index focusing upon economic metrics, represents another tool useful in quantifying disparities between rural and non-rural communities across the US (Khatana et al., 2019). This composite index is composed of the following variables: no high school diploma, poverty rate, adults not working, housing vacancy rate, median household income, change in employment, and change in establishments (Economic Innovation Group, 2018). The most recent available county-level DCI data (encompassing 2012-2016) expressed as a nationally indexed ranked score ranging from 0 (best performing county) to 100 (worst performing) was included in an effort to better describe county-level economic health. All of these variables were assembled into a county-level database in which multivariable analysis was conducted as soon as the COVID-19 data was input on August 1, 2020.

\section{Statistical Methods}

All statistical analysis was performed using SAS 9.4 and statistical significance was assessed using an alpha level of 0.05. Descriptive statistics on all variables were determined and included frequencies and percentages for categorical variables and means and standard deviations for continuous variables. Generalized linear regression models assuming a Poisson distribution and log link and accounting for overdispersion by deviance scaling were used to examine the association of county level demographic, DCI, SVI theme, cardiovascular death rates, and diabetes percentages with COVID-19 cumulative incidence and cumulative case-fatality rates. All continuous variables were centered to their mean and the centered variables were used in modeling. Georgia-based SVI rankings for SES, household, minority, and housing and transportation themes were categorized into quartiles. All variables were examined for potential multicollinearity before modeling using variance inflation factors. All variance inflation factors were less than 5 indicating no multicollinearity between variables. Simple bivariate models were first examined for each individual independent variable. Then a full multivariable model was created and a backward model building strategy was used to arrive at a final multivariable model for each outcome. Non-significant variables were removed from each model one at a time and model fit criteria including the scaled Pearson chi-square, Akaike's Information Criterion, and Bayes Information Criterion. If all three fit statistics decreased the variable remained out of the model, otherwise the variable was placed back into the model and the next non-significant variable was removed. For each outcome, the final model consisted of main effects or interaction terms that were statistically significant or needed in the model to improve the model fit criteria. The estimated risk ratio (RR) for COVID-19 cumulative incidence and cumulative case fatality and corresponding 95\% CI were determined for each independent variable: non-rural vs rural; each SVI quartile vs the lowest quartile; a 5\% increase for the distressed communities index, and percent with diabetes; and a 100/100,000 increase in coronary heart disease, heart failure, hypertension, stroke, and cardiac dysrhythmia death rates.

\section{RESULTS}

\section{COVID-19 Incidence - Poisson Regression Modeling Results}

In simple, bivariate models (Table 1), all variables except cardiac dysrhythmia death rates were significantly associated with COVID-19 incidence rates. Increased risk of COVID-19 was seen in non-rural compared to rural counties. Likewise, those with the highest and second highest SES vulnerability, the highest and second highest household vulnerability, the second lowest minority vulnerability, and the highest and second lowest housing and transportation vulnerability were at risk for incident COVID-19. Risk of COVID-19 was higher in communities with higher distress community indexes. Finally increasing coronary heart disease, heart failure, hypertension, and 
Table 1

Descriptive Statistics and Simple Poisson Regression Results on Georgia Counties

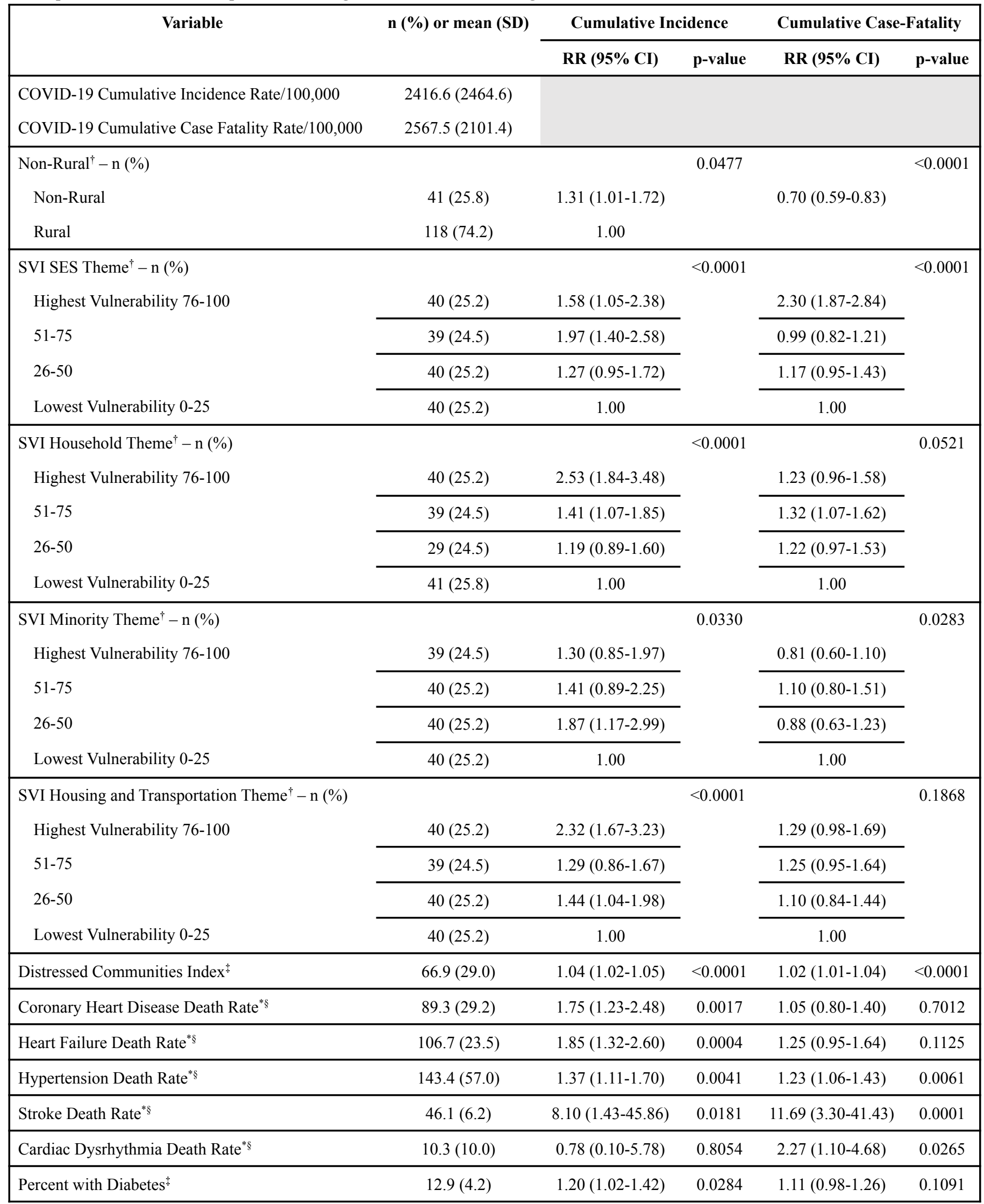

Rate/100,000, "Estimated RR are for a 1-unit increase in the independent variable, ${ }^{\star}$ Estimated RR are for a 5\% increase in the independent variable, ${ }^{\S}$ Estimated RR are for a 100/100,000 increase in the independent variable. SVI = Social Vulnerability Index; SES $=$ Socioeconomic Status, $\mathrm{RR}=$ Risk Ratio. 
stroke death rates and increasing diabetes prevalence were associated with increased COVID-19 infection at the county level.

The final multivariable model (Table 2) contained non-rural status, each SVI theme, and heart failure death rates. Counties that were non-rural, in the highest SES vulnerability group, in the highest household vulnerability group, in the second lowest minority vulnerability group, in the highest housing and transportation vulnerability group, and who had higher heart failure death rates had increased risk of COVID-19 incidence.

\section{COVID-19 Case-Fatality Rate - Multivariable Modeling Results}

In simple, bivariate models (Table 1), non-rural counties had lower risk of cumulative case-fatality rates. Higher risk of cumulative case-fatality rates was noted in the highest SES vulnerability group and the second highest household vulnerability group. Higher distress indexes and higher hypertension, stroke, and cardiac dysrhythmia death rates were also found to have an increased risk in simple, bivariate models.

In the final multivariable model (Table 2), non-rurality was not significantly associated with COVID-19 case-fatality rates. Counties with the highest SES vulnerability, the second highest and second lowest household vulnerability, the second highest and second lowest housing and transportation vulnerability, and counties with higher stroke death rates were at increased risk of case-fatality. Counties with the highest minority vulnerability and increasing coronary heart disease death rates had lower risk of case-fatality.

Table 2

Multivariable Poisson Regression Models for COVID-19 Cumulative Incidence and Case Fatality Rates in Georgia

\begin{tabular}{|c|c|c|c|c|c|}
\hline \multirow[b]{2}{*}{ Effect in Model } & \multirow[b]{2}{*}{ Level } & \multicolumn{2}{|c|}{$\begin{array}{l}\text { COVID-19 Cumulative } \\
\text { Incidence }\end{array}$} & \multicolumn{2}{|c|}{$\begin{array}{l}\text { COVID-19 Cumulative } \\
\text { Case Fatality }\end{array}$} \\
\hline & & RR $(95 \% C I)$ & p-value & RR $(95 \% C I)$ & p-value \\
\hline Non-Rural $^{\dagger}$ & Non-Rural vs Rural & $1.98(1.52-2.58)$ & $<0.0001$ & & \\
\hline \multirow[t]{3}{*}{ SVI SES Theme ${ }^{\dagger}$} & Highest 76-100 vs Lowest Vulnerability 0-25 & $1.09(0.64-1.88)$ & 0.0200 & $1.54(1.10-2.16)$ & $<0.0001$ \\
\hline & $51-75$ vs Lowest Vulnerability $0-25$ & $1.24(0.83-1.87)$ & & $0.73(0.52-1.01)$ & \\
\hline & $26-50$ vs Lowest Vulnerability $0-25$ & $0.75(0.51-1.11)$ & & $0.92(0.69-1.24)$ & \\
\hline \multirow[t]{3}{*}{ SVI Household Theme ${ }^{\dagger}$} & Highest 76-100 vs Lowest Vulnerability 0-25 & $2.90(1.97-4.28)$ & $<0.0001$ & $1.24(0.92-1.68)$ & 0.0981 \\
\hline & $51-75$ vs Lowest Vulnerability $0-25$ & $1.10(0.78-1.56)$ & & $1.32(1.03-1.71)$ & \\
\hline & $26-50$ vs Lowest Vulnerability $0-25$ & $1.19(0.91-1.56)$ & & $1.27(1.02-1.59)$ & \\
\hline \multirow[t]{3}{*}{ SVI Minority Theme ${ }^{\dagger}$} & Highest 76-100 vs Lowest Vulnerability 0-25 & $0.95(0.65-1.40)$ & 0.0661 & $0.66(0.49-0.89)$ & 0.0161 \\
\hline & $51-75$ vs Lowest Vulnerability $0-25$ & $0.86(0.58-1.29)$ & & $0.86(0.64-1.16)$ & \\
\hline & 26-50 vs Lowest Vulnerability 0-25 & $1.29(0.87-1.90)$ & & $0.80(0.59-1.08)$ & \\
\hline \multirow{3}{*}{$\begin{array}{l}\text { SVI Housing and Transportation } \\
\text { Theme }^{\dagger}\end{array}$} & Highest $76-100$ vs Lowest Vulnerability 0-25 & $2.04(1.33-3.13)$ & 0.0015 & $1.38(0.99-1.93)$ & 0.0413 \\
\hline & $51-75$ vs Lowest Vulnerability $0-25$ & $1.29(0.91-1.84)$ & & $1.52(1.14-2.03)$ & \\
\hline & $26-50$ vs Lowest Vulnerability $0-25$ & $1.51(1.12-2.02)$ & & $1.31(1.03-1.68)$ & \\
\hline $\begin{array}{l}\text { Coronary Heart Disease Death } \\
\text { Rate }^{\S}\end{array}$ & & & & $0.65(0.46-0.92)$ & 0.0153 \\
\hline Heart Failure Death Rate ${ }^{\S}$ & & $1.41(0.95-2.08)$ & 0.0877 & & \\
\hline Stroke Death Rate ${ }^{\S}$ & & & & $8.46(2.36-30.22)$ & 0.0010 \\
\hline
\end{tabular}

'Estimated RR are for a 1-unit increase in the independent variable, ${ }^{\dagger}$ Estimated RR are for a 5\% increase in the independent variable, ${ }^{\S}$ Estimated $\mathrm{RR}$ are for a 10/100,000 increase in the independent variable. SVI $=$ Social Vulnerability Index; SES $=$ Socioeconomic Status, RR $=$ Risk Ratio. 


\section{DISCUSSION}

Our multivariable regression modeling results indicate that through the beginning of August 2020, non-rural counties in Georgia exhibited significantly higher COVID-19 incidence rates $[\mathrm{RR}=1.98 ; 95 \% \mathrm{CI}(1.52,2.58)]$. This finding may be the result of disparities in access to COVID-19 testing across the state since the onset of the pandemic. As the pandemic progresses nationwide, further investigation into the relationship between rurality and COVID-19 epidemiology will likely remain a topic of significant investigation.

Our analysis also revealed the association between higher rates of coronary heart disease burden and lower COVID-19 case-fatality (Table 2). Using CDC-defined categories of heart failure mortality rates at the county level reveals higher COVID-19 incidence rates for counties with higher rates of nearly all forms of recent, county-level heart failure related mortality. COVID-19 case-fatality rates were most strongly associated with counties demonstrating historically higher rates of stroke $[\mathrm{RR}=8.46 ; 95 \% \mathrm{CI}(2.36,30.22)]$.

Holman et al (2020) recently reported worse outcomes in both type 1 and 2 diabetic patients with a history of stroke affected by COVID-19. The relationship between historical stroke rates and worsened COVID-19 incidence and outcomes may be related to the underlying relationship between stroke and rurality. The CANHEART stroke study published in 2019 revealed an adverse association between rural status and stroke rates and fatality in Canada (Kapral et al., 2019). In our multivariable model examining COVID-19 case-fatality, however, rural county status fell out of the adjusted model while county level stroke mortality rates remained (Table 2). This suggests that COVID-19 mortality may be less influenced by geographic factors than underlying disease burden characteristics across Georgia. Due to the high burden of stroke across Georgia and the southeastern United States, this finding warrants further investigation in future studies.

As has been described in prior studies, poor socioeconomic characteristics appear to be associated with worse COVID-19 outcomes globally (Ahmed et al., 2020; de Souza et al., 2020; Singu et al., 2020). Our data support these international findings, but at the county level in Georgia. The component themes of the SVI examine the impact of differing socioeconomic factors upon a community's social vulnerability, particularly to infrequent shocks to their economic and/or health stability. Our multivariable analysis revealed similar results when examining the impact of differing themes on COVID-19 incidence and case-fatality rates. Counties with the highest socioeconomic vulnerability (SES theme) demonstrated significantly higher risk of both COVID-19 incidence and case-fatality. Mixed results were observed for other SVI themes including household and minority themes (Table 2). Intriguingly, counties with the highest minority vulnerability index were found to have a significantly lower COVID-19 case-fatality rate. In contrast, Richmond and colleagues reported the percentage of non-Hispanic white residents as being the most protective variable in predicting COVID-19 case rates in their Georgia county-level analysis (Richmond et al., 2020). These differing findings regarding the epidemiology of COVID-19 and minority status across Georgia could be accounted for by the different composite variables that make up the Robert Woods Johnson Foundation's County Health Rankings utilized in Richmond's analysis, and the CDC's SVI data used in this study. Richmond et al did observe similar trends of low socioeconomic status markers such as percent of children living in poverty and percent of children qualifying for free lunch being positively associated with COVID-19 cumulative case rates. Their results highlight differing study designs between their analysis and ours, potentially owing to the different sources and composition of the socioeconomic data included in each analysis.

Another metric of county-level economic vulnerability, the DCI, was associated with higher COVID-19 incidence and case-fatality rates in univariate analysis. However, the DCI failed to remain in the final adjusted model, perhaps owing to DCI's focus on variables more purely economic in nature. It is important to note that the SVI's socioeconomic, household, and transportation themes did remain significant in our adjusted findings, emphasizing that COVID-19 cumulative incidence and case fatality rates are observed to be worse in more socially vulnerable counties in Georgia.

An unexpected but intriguing additional finding was that counties with historically higher rates of cardiovascular death attributable to coronary heart disease (CHD) were associated with a decreased risk of COVID-19 cumulative case fatality rates $[R R=0.6595 \%$ CI $(0.46-0.92)]$. A hypothetical, yet untested, explanation for this protective association between CHD mortality and COVID-19 mortality rates would be that of statin use. Statins, a class of lipid lowering agents indicated for chronic administration in patients with CHD, have been reportedly associated with improved COVID-19 outcomes in several studies (Castiglione et al., 2020; Kow and Hasan, 2020; Stone et al., 2014; Zhang et al., 2020). Statin use has been shown to vary geographically and across county lines. Counties with greater racial and socioeconomic disparities, particularly those with lower health insurance coverage and higher proportions of Medicare Part D low-income subsidies were associated with decreased statin use (Karpinski et al., 2019; Schroff et al., 2017). While conjectural, further investigation regarding the role of statin use for the treatment and prevention of COVID-19 remains a topic of investigation at an international scale.

\section{Limitations}

This study has several limitations. COVID-19 incidence and case-fatality data have changed in Georgia since the beginning of August 2020. Additionally, numerous other socioeconomic and chronic health variables could be included in this type of analysis. However, we sought to utilize widely accepted indices of community vulnerability and distress that are indexed nationally rather than create a portfolio of independent variables ourselves. Additionally, 
our background in cardiovascular medicine and clinical observations of worse outcomes in patients with underlying cardiovascular disease in Georgia led to our inclusion of $\mathrm{CDC}$ data in this analysis.

While compiling population data from the county level provided the opportunity for large-scale assessment, there is the risk of aggregation bias and ecological fallacy when extrapolating these results to the individual level. However, county-level data was utilized in this analysis owing to the availability of well accepted, nationally available socioeconomic and clinical population-level data across Georgia at the county level. Further exploratory work could consider the role of other chronic diseases such as pulmonary conditions or cancer and their impact on COVID-19 outcomes.

\section{CONCLUSIONS}

Rural county status in the state of Georgia is associated with a lower risk of COVID-19 incidence when compared to non-rural counties through the beginning of August 2020. Despite a lower observed incidence in rural counties, we detected no significant difference in COVID-19 fatality rates between rural and non-rural counties in adjusted multivariable modeling. These findings may be related to disparities not only in access to COVID-19 testing, but clinical care as well. Worsened indices of socioeconomic vulnerability and higher historical rates of stroke mortality at the county-level are associated with poorer COVID-19 outcomes. Further investigation into the relationship between preexisting cardiovascular disease burden, particularly stroke, and COVID-19 outcomes appears warranted.

\section{References}

Ahmed, F., Ahmed, N., Pissarides, C., \& Stiglitz, J. (2020). Why inequality could spread COVID-19. The Lancet: Public Health, 5(5), e240. https://doi.org/10.1016/S2468-2667(20)30085-2

Bansal M. (2020). Cardiovascular disease and COVID-19. Diabetes \& Metabolic Syndrome, 14(3), 247-250. https://doi.org/10.1016/j.dsx.2020.03.013

Castiglione, V., Chiriacò, M., Emdin, M., Taddei, S., \& Vergaro, G. (2020). Statin therapy in COVID-19 infection. European Heart Journal: Cardiovascular Pharmacotherapy, 6(4), 258-259. https://doi.org/10.1093/ehjcvp/pvaa042

CDC's Social Vulnerability Index. (2018, September 12). Retrieved July 10, 2020, from https://svi.cdc.gov/index.html

COVID-19 Status Report. (2020). Georgia Department of Public Health. Retrieved August 1, 2020, from https://dph.georgia.gov/covid19-daily-status-report

de Souza, C., Machado, M. F., \& do Carmo, R. F. (2020). Human development, social vulnerability and COVID-19 in Brazil: a study of the social determinants of health. Infectious Diseases of Poverty. 9(1), 124. https://doi.org/10.1186/s40249-020-00743-x

From Great Recession to Great Reshuffling: Charting a Decade of Change Across American Communities. (October 2018). Economic Innovation Group. Retrieved July 10, 2020, from https://eig.org/wp-content/uploads/2018/10/2018-DCI.pdf.
Georgia Department of Public Health Daily Status Report. (2020). Georgia Department of Public Health. Retrieved August 1, 2020, from https://dph.georgia.gov/covid-19-daily-status-report.

Georgia Department of Public Health OASIS. (2020). Georgia Department of Public Health. Retrieved August 1, 2020, from https://oasis.state.ga.us.

Guzik, T. J., Mohiddin, S. A., Dimarco, A., Patel, V., Savvatis, K., Marelli-Berg, F. M., Madhur, M. S., Tomaszewski, M., Maffia, P., D'Acquisto, F., Nicklin, S. A., Marian, A. J., Nosalski, R., Murray, E. C., Guzik, B., Berry, C., Touyz, R. M., Kreutz, R., Wang, D. W., Bhella, D., McInnes, I. B. (2020). COVID-19 and the cardiovascular system: implications for risk assessment, diagnosis, and treatment options. Cardiovascular Research. 116(10), 1666-1687. https://doi.org/10.1093/cvr/cvaa106.

Henning-Smith, C., Tuttle, M., \& Kozhimannil, K. B. (2020). Unequal Distribution of COVID-19 Risk Among Rural Residents by Race and Ethnicity. The Journal of Rural Health. 10.1111/jrh.12463. Advance online publication. https://doi.org/10.1111/jrh.12463

Holman, N., Knighton, P., Kar, P., O'Keefe, J., Curley, M., Weaver, A., Barron, E., Bakhai, C., Khunti, K., Wareham, N. J., Sattar, N., Young, B., \& Valabhji, J. (2020). Risk factors for COVID-19-related mortality in people with type 1 and type 2 diabetes in England: a population-based cohort study. The lancet. Diabetes \& endocrinology, 8(10), 823-833. https://doi.org/10.1016/S2213-8587(20)30271-0

Holuka, C., Merz, M. P., Fernandes, S. B., Charalambous, E. G., Seal, S. V., Grova, N., \& Turner, J. D. (2020). The COVID-19 Pandemic: Does Our Early Life Environment, Life Trajectory and Socioeconomic Status Determine Disease Susceptibility and Severity? International Journal of Molecular Sciences, 21(14), 5094. https://doi.org/10.3390/ijms21145094

Huertas, A., Montani, D., Savale, L., Pichon, J., Tu, L., Parent, F., Guignabert, C., \& Humbert, M. (2020). Endothelial cell dysfunction: a major player in SARS-CoV-2 infection (COVID-19)? The European Respiratory Journal, 56(1), 2001634. https://doi.org/10.1183/13993003.01634-2020

Interactive Atlas of Heart Disease and Stroke. (2020). Center for Disease Control and Prevention. Retrieved July 10, 2020 from: https://nccd.cdc.gov/DHDSPAtlas/?state=County.

Kapral, M. K., Austin, P. C., Jeyakumar, G., Hall, R., Chu, A., Khan, A. M., Jin, A. Y., Martin, C., Manuel, D., Silver, F. L., Swartz, R. H., \& Tu, J. V. (2019). Rural-Urban Differences in Stroke Risk Factors, Incidence, and Mortality in People With and Without Prior Stroke. Circulation. Cardiovascular Quality and Outcomes, 12(2), e004973. https://doi.org/10.1161/CIRCOUTCOMES.118.004973

Karaye, I. M., \& Horney, J. A. (2020). The Impact of Social Vulnerability on COVID-19 in the U.S.: An Analysis of Spatially Varying Relationships. American Journal of Preventive Medicine. 59(3), 317-325. https://doi.org/10.1016/j.amepre.2020.06.006

Karpinski, S. A., Look, K. A., \& Vanness, D. J. (2019). Factors Associated with County-Level Variation in the Prescription of Statins. Journal of managed care \& specialty pharmacy, 25(12), 1358-1365. https://doi.org/10.18553/jmcp.2019.25.12.1358

Kaufman, B. G., Whitaker, R., Pink, G., \& Holmes, G. M. (2020). Half of Rural Residents at High Risk of Serious Illness Due to COVID-19, Creating Stress on Rural Hospitals. The 
Journal of Rural Health. 36(4), 584-590.

https://doi.org/10.1111/jrh.12481

Khatana, SAM., Nathan, A., Dayoub, EJ., Khazi, D., Yeh, R., Groeneveld, P., Subramanian, S. (2019) Trends in Cardiovascular Mortality and Economic Distress in Communities. Circulation. 2019;140(Suppl_1):A12671-A12671.

Kow, C. S., \& Hasan, S. S. (2020). Meta-analysis of Effect of Statins in Patients with COVID-19. The American Journal of Cardiology, 134, 153-155. https://doi.org/10.1016/j.amjcard.2020.08.004

Lakhani, H. V., Pillai, S. S., Zehra, M., Sharma, I., \& Sodhi, K. (2020). Systematic Review of Clinical Insights into Novel Coronavirus (CoVID-19) Pandemic: Persisting Challenges in U.S. Rural Population. International Journal of Environmental Research and Public Health. 17(12), 4279. https://doi.org/10.3390/ijerph17124279

Madjid, M., Safavi-Naeini, P., Solomon, S. D., \& Vardeny, O. (2020). Potential Effects of Coronaviruses on the Cardiovascular System: A Review. JAMA Cardiology. 5(7), 831-840. https://doi.org/10.1001/jamacardio.2020.1286

Paramasivam, A., Priyadharsini, J. V., Raghunandhakumar, S., \& Elumalai, P. (2020). A novel COVID-19 and its effects on cardiovascular disease. Hypertension Research. 43(7), 729-730. https://doi.org/10.1038/s41440-020-0461-x

Peters D. J. (2020). Community Susceptibility and Resiliency to COVID-19 Across the Rural-Urban Continuum in the United States. The Journal of Rural Health. 36(3), 446-456. https://doi.org/10.1111/jrh.12477

Richmond, H. L., Tome, J., Rochani, H., Fung, I. C., Shah, G. H., \& Schwind, J. S. (2020). The Use of Penalized Regression Analysis to Identify County-Level Demographic and Socioeconomic Variables Predictive of Increased COVID-19 Cumulative Case Rates in the State of Georgia. International Journal of Environmental Research and Public Health. 17(21), 8036. https://doi.org/10.3390/ijerph17218036

Schroff, P., Gamboa, C. M., Durant, R. W., Oikeh, A., Richman, J. S., \& Safford, M. M. (2017). Vulnerabilities to Health Disparities and Statin Use in the REGARDS (Reasons for Geographic and Racial Differences in Stroke) Study. Journal of the American Heart Association, 6(9), e005449.

Singu, S., Acharya, A., Challagundla, K., \& Byrareddy, S. N. (2020). Impact of Social Determinants of Health on the Emerging COVID-19 Pandemic in the United
States. Frontiers in Public Health, 8, 406.

https://doi.org/10.3389/fpubh.2020.00406

Souch, J. M., \& Cossman, J. S. (2020). A Commentary on Rural-Urban Disparities in COVID-19 Testing Rates per 100,000 and Risk Factors. The Journal of Rural Health. 10.1111/jrh.12450. Advance online publication. https://doi.org/10.1111/jrh.12450

Stone, N. J., Robinson, J. G., Lichtenstein, A. H., Bairey Merz, C. N., Blum, C. B., Eckel, R. H., Goldberg, A. C., Gordon, D., Levy, D., Lloyd-Jones, D. M., McBride, P., Schwartz, J. S., Shero, S. T., Smith, S. C., Jr, Watson, K., Wilson, P. W., \& American College of Cardiology/American Heart Association Task Force on Practice Guidelines (2014). 2013 ACC/AHA guideline on the treatment of blood cholesterol to reduce atherosclerotic cardiovascular risk in adults: a report of the American College of Cardiology/American Heart Association Task Force on Practice Guidelines. Journal of the American College of Cardiology, 63(25 Pt B), 2889-2934. https://doi.org/10.1016/j.jacc.2013.11.002

Varga, Z., Flammer, A. J., Steiger, P., Haberecker, M., Andermatt, R., Zinkernagel, A. S., Mehra, M. R., Schuepbach, R. A., Ruschitzka, F., \& Moch, H. (2020). Endothelial cell infection and endotheliitis in COVID-19. Lancet. 395(10234), 1417-1418. https://doi.org/10.1016/S0140-6736(20)30937-5

Wang, D., Hu, B., Hu, C., Zhu, F., Liu, X., Zhang, J., Wang, B., Xiang, H., Cheng, Z., Xiong, Y., Zhao, Y., Li, Y., Wang, X., \& Peng, Z. (2020). Clinical Characteristics of 138 Hospitalized Patients With 2019 Novel Coronavirus-Infected Pneumonia in Wuhan, China. JAMA, 323(11), 1061-1069. https://doi.org/10.1001/jama.2020.1585

Zhang, X. J., Qin, J. J., Cheng, X., Shen, L., Zhao, Y. C., Yuan, Y., Lei, F., Chen, M. M., Yang, H., Bai, L., Song, X., Lin, L., Xia, M., Zhou, F., Zhou, J., She, Z. G., Zhu, L., Ma, X., Xu, Q., Ye, P., ... Li, H. (2020). In-Hospital Use of Statins Is Associated with a Reduced Risk of Mortality among Individuals with COVID-19. Cell Metabolism. 32(2), 176-187.e4. https://doi.org/10.1016/j.cmet.2020.06.015

Zheng, Y. Y., Ma, Y. T., Zhang, J. Y., \& Xie, X. (2020). COVID-19 and the cardiovascular system. Nature Reviews: Cardiology. 17(5), 259-260. https://doi.org/10.1038/s41569-020-0360-5

(C) Michael Morgan, Lavannya Atri, Jennifer L. Waller, D. Douglas Miller, and Adam E. Berman. Originally published in jGPHA

(http://www.gapha.org/igpha/) May 15, 2021. This is an open-access article distributed under the terms of the Creative Commons Attribution Non-Commercial No-Derivatives License (http://creativecommons.org/licenses/by/4.0/), which permits unrestricted use, distribution, and reproduction in any medium, provided the original work ("first published in the Journal of the Georgia Public Health Association...") is properly cited with original URL and bibliographic citation information. The complete bibliographic information, a link to the original publication on http://www.gapha.jgpha.org/, as well as this copyright and license information must be included. 